\title{
Response inhibition under task switching: its strength depends on the amount of task-irrelevant response activation
}

\author{
Michel D. Druey Ronald Hübner
}

\begin{abstract}
Under task switch conditions, response repetitions usually produce benefits if the task also repeats, but costs if the task switches. So far, it is largely undecided how to account for these effects. In the present study, we provide additional evidence in favor of the account that each response is inhibited in order to prevent its accidental re-execution. To test this hypothesis, the risk of an accidental re-execution of a given response was manipulated by modulating the activation of the response in the previous task. In Experiment 1, this was done by means of congruent and incongruent stimuli. As expected, on task switch trials, the repetition costs were larger if a congruent rather than an incongruent stimulus occurred in the previous task. In Experiment 2, the same effect occurred for stimulusresponse compatible versus incompatible stimuli in the previous task. In Experiment 3, both manipulations were applied together, which produced almost additive effects. Altogether, the results support the inhibition account for the response repetition effects under task switch conditions.
\end{abstract}

\section{Introduction}

One of the central issues in psychology concerns how perception and action are related. In order to investigate this question, participants are usually asked to perform simple reactive tasks, in which they have to respond to stimuli according to a pre-specified stimulus-response (SR) mapping as fast and as accurately as possible. From the observed effects in response time and error rate, one can

M. D. Druey ( $\measuredangle)$ - R. Hübner

Universität Konstanz, Fachbereich Psychologie,

Fach D29, 78457 Konstanz, Germany

e-mail: michel.druey@uni-konstanz.de then infer which mental processes and representations are involved in performing these tasks.

One type of information that has been considered as valuable in this respect are response repetition effects (e.g. Campbell \& Proctor, 1993; Pashler \& Baylis, 1991; Smith, 1968; Soetens, 1998). Usually, responses repetitions produce an advantage compared to response shifts. Recent results from task switch studies, however, revealed a more complex picture. Rogers and Monsell (1995), for instance, also found benefits for response repetitions, but only when the task repeated as well. On task switch trials, response repetitions produced costs. Meanwhile, this interaction between task switching and response repetition has been replicated in several studies (e.g., Mayr \& Kliegl, 2003; Meiran, Chorev, \& Sapir, 2000). To explain this interaction, several accounts have been proposed, which can be classified into three types based on a suggestion by Rogers and Monsell (1995; see Hübner \& Druey, 2006 for a more detailed overview). First, it is conceivable that the effects are due to the modulation of the association strengths between stimulus categories and responses (e.g., "even" number-"left" response) (e.g., Meiran, 2000a; Meiran et al., 2000; Schuch \& Koch, 2004). Second, the effects could also be caused by response (re-) reconfiguration as one component of a general task-set reconfiguration mechanism (e.g. Kleinsorge, 1999). A third possible mechanism could be response inhibition. In our previous study (Hübner \& Druey, 2006), the best account to explain the pattern of our results was response inhibition. Therefore, we examined this account in more detail in the present study.

According to the response inhibition account there is a general tendency to inhibit the last response in order to prevent its accidental re-execution. Consequently, if this last response is required again, it is still in an inhibited state, which slows responding relative to when an alterna- 
tive response is required. The fact that costs are observed only on task switch trials can be explained by taking stimulus category repetitions into account. Obviously, on task repetition trials, on which the last response is required again, the previously relevant stimulus category also repeats. Consequently, the residual activation of the stimulus category (and/or a strengthened association between the stimulus category and the response (category); we will return to this issue in the "General discussion") outweighs the negative effects of response inhibition. Thus, any inhibition-based explanation of the repetition effects under task switching essentially requires the assumption of two mechanisms: (a) response inhibition as the core process, and (b) stimulus category priming as possible compensating mechanism on task repetition trials. Furthermore, the combination of these mechanisms also explains why costs have also been observed in several previous studies, in which a constant task but non-classifiable stimuli had been used (e.g., Campbell \& Proctor, 1993; Pashler \& Baylis, 1991; Smith, 1968).

A further important aspect with respect to the response repetition effects under task switching is the valence of the stimuli. In most of the studies, either univalent or bivalent stimuli were used. Whereas univalent stimuli can be evaluated only with respect to the currently required task, and not with respect to the currently irrelevant task, bivalent stimuli can be evaluated according to both tasks (i.e. with respect to the currently relevant and irrelevant task). In our previous study, we observed that response repetition effects were relatively small for univalent stimuli in contrast to bivalent stimuli (Hübner \& Druey, 2006).

An important further characteristic of bivalent stimuli is that they can be congruent or incongruent. This means that a given stimulus might activate the same response according to both possible tasks (i.e. the currently relevant and the currently irrelevant one: congruent stimulus), or it might activate competing responses (incongruent stimulus). Consequently, the risk of accidental response re-executions is increased especially with incongruent (bivalent) stimuli since they may result in an activation of the response just produced on the previous trial, although this response is not the currently required one. In order to avoid accidental response re-executions, we therefore hypothesized that the strength of response inhibition increases with the risk of an accidental response re-execution (Hübner \& Druey, 2006).

To test this hypothesis, we examined how the congruency on a given trial affected the size of the response repetition effects on the subsequent trial ${ }^{1}$ in our previous study

\footnotetext{
"Here and in the following, we use the term "trial" even if actually effects within a trial (i.e. from a first to a second task in a dual-task or sequence procedure) were considered.
}

(see Brown, Reynolds, \& Braver, 2007 for a similar reasoning). The idea was that some of the response activation on a given trial is usually carried over to the next trial. Consequently we assumed that the higher the activation of the required response on the previous trial, or the higher the activation difference between the required and the alternative response, the higher the risk that it will erroneously be re-executed on the present trial. Furthermore, we assumed that congruent stimuli either produce larger activation of the required response than incongruent stimuli, or that they result in a larger activation difference between the required and the alternative response. Both of these assumptionswhich-are also not mutually exclusive-seem reasonable from several parallel distributed processing (PDP) models, which were developed to explain the performance in tasks, in which response competition occurs due to the simultaneous presentation of target and distractor stimuli (e.g. Botvinick, Braver, Barch, Carter, \& Cohen, 2001; Brown et al., 2007; Gilbert \& Shallice, 2002; Houghton \& Tipper, 1994). Furthermore, in these models, the activation of the (relevant) response node increases further even after the response threshold was reached. This increase in activation is larger for congruent than for incongruent stimuli due to stronger lateral inhibition in the latter case.

From these assumptions it is also clear why congruent stimuli produce faster responses than incongruent stimuli. Moreover, given the stronger (relative) response activation for congruent than for incongruent stimuli, we supposed that stronger inhibition is also required after congruent than after incongruent stimuli in order to prevent that the same response is erroneously re-executed. Consequently, according to the inhibition account, the response repetition costs should be larger if, on the previous trial, a congruent rather than an incongruent stimulus appeared. This was exactly what we observed (Hubner \& Druey, 2006). Likewise, on task repetition trials, a congruent stimulus on the previous trial led to reduced response repetition benefits, compared to incongruent stimuli. Thus, taken together, these effects can easily be explained by the assumption that the degree of response inhibition is determined by the amount of previous response activation. If one further assumes that inhibition takes some time to build up after the activation of a response (Houghton \& Tipper, 1994; Ridderinkhof, 2002), then the activation of the response at the time of response selection might not be relevant for determining the strength of response inhibition, but rather the activation at the time when response inhibition is triggered.

A further interesting aspect in our previous experiments was that we observed repetition effects even though there were no literal response repetitions (Hübner \& Druey, 2006; see also Schuch \& Koch, 2004). We used a task switch paradigm that was combined with a dual-task procedure, 
where the participants always responded to a first stimulus with the left hand and to a second stimulus with the right hand by pressing one of two horizontally arranged response buttons, respectively. Thus, because the participants had to respond to each task with an individual hand, there were response category (left, right) repetitions rather than literal response repetitions. Nevertheless, the repetition effects were analogous to those observed for true response repetitions. Thus, for simplicity, we will again use the term "response repetition" even when actually "response category repetition" is meant.

The idea that the risk of an accidental re-execution of the last response modulates the strength of response inhibition also received support from a task switch study of Steinhauser and Hübner (2006), in which the participants had to respond very fast in order to meet a response deadline. As a result, response repetitions produced costs even on task repetition trials. This can be explained by assuming that, under time pressure, the response criterion is rather low, which increases the risk of accidental response re-executions. Therefore, response inhibition must be rather strong under such conditions in order to limit the number of erroneous response re-executions.

Altogether, the so far mentioned studies support the hypothesis that the strength of response inhibition is determined by the risk of accidentally re-executing the last response: The higher the risk, the stronger the inhibition. Moreover, it does not seem to matter whether the risk is increased by a lowered response criterion, by response competition, or by stronger (relative) response activation.

In the present study, we further investigated the effects of response activation on the strength of response inhibition and the repetition effects under task switching. As mentioned, in our former study we modulated response activation by varying the congruency of the stimuli (Huibner \& Druey, 2006). The main question in the present study was to what extent this manipulation was specific. Although, the additional response activation contributed by congruent stimuli was produced by a stimulus feature that was currently task irrelevant, it nevertheless was generally task relevant within a given block of trials. Thus, one could ask whether the same effects on response inhibition can also be obtained by increasing response activation via a completely task irrelevant feature. Moreover, it was examined whether the response repetition effects can simultaneously be modulated by multiple sources. Therefore, in Experiment 1, we again modulated response activation by stimulus congruency. In Experiment 2, response activation was then varied by means of spatial stimulusresponse (SR-) compatibility (cf. Simon, 1969; Hommel, 1993; see, e.g., Lu \& Proctor, 1995, for an overview). Finally, in Experiment 3 both manipulations were applied together.

\section{Experiment 1}

The first experiment should replicate our former results with a slightly modified procedure. Furthermore, it should serve as a baseline for the subsequent experiments. In the present experiment, the participants had to perform two tasks on each trial. However, in contrast to our previous study (Hibbner \& Druey, 2006), here, there was no temporal overlap between the first and the second task. Moreover, task repetition and task switch trials were randomized within a block, and a pre-cue indicated the trial type. With these modifications, our procedure was more similar to that in standard task switch studies. At the same time, we again used trials with two tasks in order to prevent higher-order repetition effects (cf. Soetens, 1998), i.e. repetition effects accumulating over the run of several trials. In other words, since we were interested in so-called "first-order repetition effects" (i.e. from one trial to the next, Soetens, 1998; Soetens, Melis, \& Notebaert, 2004), we chose a procedure in which the influence of trials prior to the last one is minimized.

The main question for the present experiment was whether, given such a procedure, the congruency of the first stimulus has similar consequences with respect to the response repetition effects as in our previous study. If this is the case, then, on task switch trials we should observe larger response repetition costs for congruent than for incongruent first stimuli. Likewise, on task repetition trials, the response repetition benefits should be reduced for congruent first stimuli.

\section{Method}

\section{Participants}

Twelve students (eight female, four male) participated in this experiment either for fulfillment of course requirements or for getting paid $(5 € / \mathrm{h})$. Their age ranged from 21 to 27 years $(M=23.2)$ and all reported normal or corrected to normal vision. Ten participants were right-handed and 2 left-handed by self-report.

\section{Apparatus and stimuli}

Stimulus presentation and response recording were controlled by an IBM-compatible PC. The stimuli were presented on a $21^{\prime \prime}$ color monitor with a resolution of $1,280 \times 768$ pixels and a refresh rate of $85 \mathrm{~Hz}$.

The digits $1,2,3,4,6,7,8$, and 9 served as stimuli. They were presented in white on black background and subtended a visual angle of $2^{\circ}$ in height and approximately $1.36^{\circ}$ in width (depending on the individual stimulus) at a viewing distance of $110 \mathrm{~cm}$. Each stimulus was presented at the center of the screen. 


\section{Procedure}

The participants had to judge the parity (odd or even) or the magnitude (less or greater than 5) of the presented numerals. Responses to the first stimulus on a trial had to be given with the left hand and responses to the second stimulus had to be given with the right hand. For each hand there was an individual response box with two horizontally arranged response buttons. The categories "even" and "less than 5" were mapped to the left response keys on each response box, and "odd" and "greater than 5" to the right response keys.

A trial started with the presentation of a cue for $900 \mathrm{~ms}$ at the center of the screen, indicating which judgment type was relevant for the first stimulus, and whether the same or the other task had to be performed with the second stimulus. The cue could have one of two forms: g/u (abbreviations of the German words "gerade" (even) and "ungerade" (odd), indicating the parity judgment), and $\mathrm{k} / \mathrm{g}$ (abbreviations of the German words "kleiner" (less) and "gröBer" (greater), indicating the magnitude judgment). Furthermore, the cue appeared in one of two colors. A green cue indicated that the same task as for the first stimulus was also required for the second stimulus. A red cue indicated that the task had to be switched from the first to the second stimulus. After the cue, a blank screen appeared for $300 \mathrm{~ms}$, followed by the first stimulus, which remained present either until the corresponding response was executed, or until the second stimulus was presented. The second stimulus always appeared $1,500 \mathrm{~ms}$ after the first stimulus and replaced the first stimulus if by this time no response to the first stimulus had been executed. Following the second response, a blank screen appeared for $2,000 \mathrm{~ms}$, before presentation of the next cue. Notice that even if the second stimulus already replaced the first one, the participants still had to execute a response to the first stimulus (and also to the second stimulus) before the next trial could start. The whole timing for a trial-sequence of two tasks is presented in Fig. 1. Participants were told to respond as fast and as accurately as possible, first to the first stimulus and then to the second stimulus.

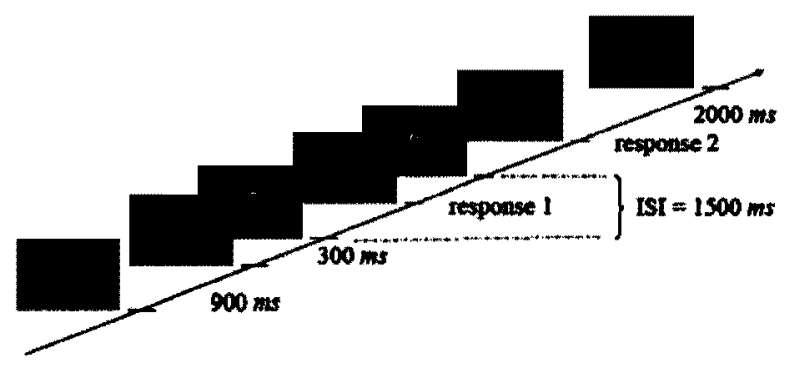

Fig. 1 Timing of presentation in the two-task sequences applied in the present experiments. ISI inter-stimulus interval
In total, the participants performed 20 blocks of 56 trials each in two 1-h sessions. The first three blocks in the first session, and the first block in the second session were considered as practice and were not analyzed.

\section{Results}

For the response times (RTs) and the error rates (ERs) to the first stimulus, the factors task (repetition, switch) and congruency (congruent, incongruent) were considered in two-way repeated measures analyses of variance (ANOVAs). The RTs and ERs for the second stimulus on a trial were analyzed by three-way ANOVAs with repeated measures on the factors task (repetition, switch), congruency of the first stimulus (congruent, incongruent), and response (repetition, shift).

\section{Responses to the first stimulus}

$R T$. The analysis revealed significant main effects of task, $F(1,11)=18.22, \quad P<0.01, \quad$ and of congruency, $F(1,11)=20.92, \quad P<0.001$. The participants responded faster on task repetition trials than on task switch trials (573 vs. $622 \mathrm{~ms}$ ). Furthermore, they responded faster to congruent stimuli than to incongruent ones (572 vs. $623 \mathrm{~ms}$ ).

$E R$. With respect to the error rates there was only a main effect of congruency, $F(1,11)=15.69, P<0.01$, reflecting fewer errors for congruent $(0.71 \%)$ than for incongruent (4.54\%) stimuli.

\section{Responses to the second stimulus}

The RTs and ERs for the second response on a trial are given in Table 1.

$R T$. The analysis revealed a significant main effect of the congruency of the first stimulus on a trial, $F(1,11)=15.55$, $P<0.01$. Responses were faster after a congruent first stimulus than after an incongruent one. Also, the main effect of response was reliable, $F(1,11)=31.23, P<0.001$, indicating response repetition costs of $18 \mathrm{~ms}$. However, there was also a two-way interaction between congruency and response, $F(1,11)=12.28, P<0.01$. As can be seen in Fig. 2 , the costs for response repetitions were larger after a

Table 1 Response times (in $m s$ ) and error rates (in \%; in parentheses) for the second response on a trial in Experiment 1, depending on task, response, and congruency of the first stimulus

\begin{tabular}{llllll}
\hline & \multicolumn{2}{l}{ Task repetition } & & \multicolumn{2}{l}{ Task switch } \\
\cline { 2 - 3 } \cline { 5 - 6 } & RCR & RCS & & RCR & RCS \\
\hline Congruent & $534(5.50)$ & $512(2.36)$ & & $584(9.56)$ & $541(4.12)$ \\
Incongruent & $528(2.98)$ & $539(2.16)$ & & $590(7.67)$ & $570(3.39)$ \\
\hline
\end{tabular}

$R C R$ response category repetition, $R C S$ response category shift 


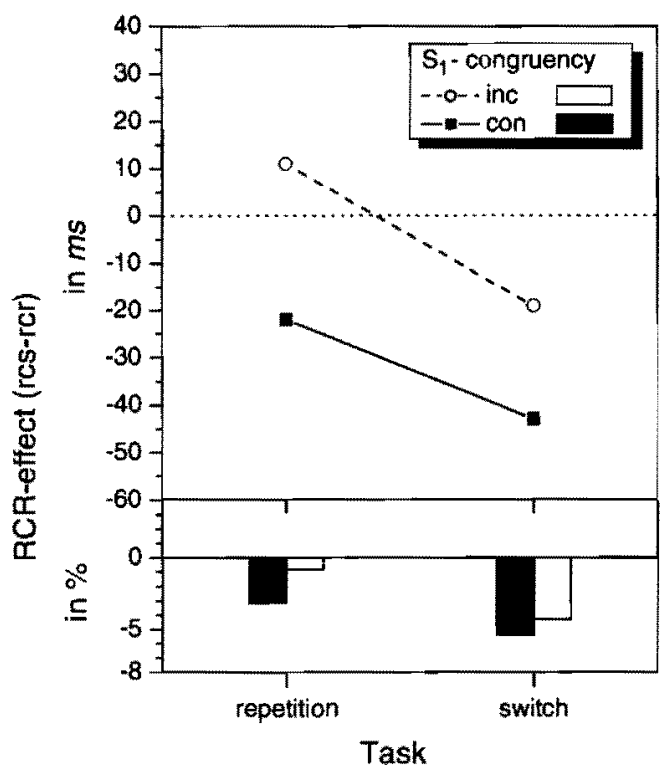

Fig. 2 Response category repetition effects, depending on task condition and congruency of the first stimulus. In the legend: con congruent, inc incongruent, $r c r$ response category repetition, $r c s$ response category shift

congruent first stimulus than after an incongruent first stimulus on task switch trials. On task repetition trials, the benefits for response repetitions after incongruent stimuli actually turned into costs after congruent first stimuli. The main effect of task was marginally significant, $F(1,11)=4.27, P=0.063$, which also holds for the interaction between task and response, $F(1,11)=4.04, P=0.070$. There was a trend in the direction that response repetition costs were larger on task switch trials $(31 \mathrm{~ms})$ than on task repetition trials $(6 \mathrm{~ms})$. The three-way interaction between task, response, and congruency was far from significant $(F<1, P>0.55)$.

$E R$. The analysis revealed a significant main effect of task, $F(1,11)=10.74, P<0.01$, reflecting switch costs of $2.93 \%$. Also, the main effect of response was significant, $F(1,11)=13.58, P<0.01$. Response repetitions produced overall costs of $3.42 \%$ relative to response shifts. The main effects of congruency of the first stimulus, $F(1,11)=4.43$, $P=0.059$, and the interaction between task and response, $F(1,11)=3.28, P=0.097$, were marginally reliable. There was a tendency towards more errors after congruent than after incongruent first stimuli, and towards larger repetition costs on task switch trials than on task repetition trials ( 4.85 vs. $1.98 \%$, respectively).

\section{Discussion}

In this experiment it was examined whether the amount of activation of the first response on a two-task trial affects the response repetition effects for the second response on that trial. As in our former study (Huibner \& Druey, 2006), the activation of the first response was modulated by the congruency of the corresponding first stimulus. That this modulation was successful can be seen from the reliable congruency effects. Responses to congruent first stimuli were substantially faster than responses to incongruent first stimuli. Moreover, as expected, the congruency of the first stimulus also affected the repetition effects for the second response. On task repetition trials, costs occurred for response repetitions if the stimulus for the first task was congruent (in the RTs and ERs), whereas benefits (in the RTs) were observed for response repetitions following an incongruent stimulus (see Fig. 2). On task switch trials, response repetitions produced costs for both stimulus conditions. However, they were larger if the first stimulus was congruent than if it was incongruent.

Because, in contrast to our former study, here there was no temporal overlap between the two tasks on a trial, the present experiment replicates and generalizes our former results. Thus, the current evidence strongly supports our hypothesis that the strength of response inhibition depends on the amount of (relative) response activation. If we consider Fig. 2, then it seems that either a stronger absolute activation of the previously relevant response, or a stronger relative activation of this response relative to the competing one generally leads to a stronger inhibition of that response, which in turn increases the latency of its re-execution on the subsequent trial. Moreover, the fact that we observed repetition costs even on task repetition trials is again strong evidence for an inhibition account of the response repetition effects under task switching. Similarly as in the study of Steinhauser and Hübner (2006), the reason might be a lowered response criterion due to speed stress in the first task of the trial. This speed stress might have been induced by using a fixed inter stimulus interval (ISI), so that the participants felt some pressure to respond to the first stimulus before the second stimulus appeared.

The results of the present experiment now serve as baseline for the following experiments. In the next one, we investigated whether it is important for the observed effects that the activation of a response is modulated by stimulus features that are related to the tasks involved in the experiment.

\section{Experiment 2}

In the first experiment we modulated the activation of the first response by the congruency of the corresponding stimulus. If the stimulus feature for the currently irrelevant task activated the same response category as the feature for the relevant task, then the (relative) activation of this response 
category was increased, compared to a situation in which the stimulus features for the two tasks activated different response categories. As a consequence, the increased (relative) response activation also led to a stronger response inhibition, which in turn increased the response repetition costs. The question for the present experiment was whether this relation between response activation and response inhibition holds generally, or whether it is restricted to certain sources of response activation.

To answer this question, we modulated the activation of the first response in a different way than in our first experiment. Here, the first stimulus on a trial was always presented laterally to the left or right of fixation. From Simontask studies (e.g, Simon, 1969; see Lu \& Proctor, 1995, for an overview) it is well known that the position of a stimulus affects performance depending on whether it is compatible or incompatible with the position of the required response. Thus, pushing a left or right response button is faster, if the stimulus appears at the left or right side, respectively. This $S R$-compatibility is effective even if the actual position of the stimulus is completely irrelevant for the task at hand. In several models, such compatibility effects are explained by assuming that the spatial features (e.g., left or right) of the stimulus directly activate the corresponding response categories (e.g., left or right) irrespective of the required SRtranslation rule (e.g., De Jong, Liang, \& Lauber, 1994; Eimer, 1995; Hommel, 1995; Kornblum, Hasbroucq, \& Osman, 1990; Ridderinkhof, 1997).

Because SR-compatibility seemed to be an efficient method to modulate response activation, it was applied in the present experiment. Moreover, in order to observe the compatibility effects in isolation, congruency effects should be excluded. Therefore, digits and letters were used as stimuli, and parity judgments and letter type (consonant/vowel) judgments were required, respectively. Because the stimuli were univalent, i.e. each stimulus type was uniquely associated with only one task, there were no congruency relations. Finally, whereas the first stimulus on a trial was presented laterally, the second stimulus always appeared centrally. This procedure was chosen to avoid effects of stimulus position repetitions and possible interactions of these effects with the response repetition effects we were interested in, which seemed likely to occur if both stimuli would have been presented laterally (cf. Hommel, 1998; Notebaert, Soetens, \& Melis, 2001; Notebaert \& Soetens, 2003; Stürmer, Leuthold, Soetens, Schroeter, \& Sommer, 2002; Stürmer, Seiss, \& Leuthold, 2005; Wühr \& Ansorge, 2005).

Thus, if the strength of response inhibition depends only on the activation of the last response, irrespective of the source of this activation, then we should observe similar effects for compatibility as we did for congruency in the first experiment. Specifically, we expected that the repeti- tion effects should generally (i.e. on task repetition and switch trials) be shifted towards (larger) costs following compatible first stimuli when compared to incompatible first stimuli.

Method

Twelve students (seven female, five male) participated in the experiment under the same conditions as in Experiment 1. Their age ranged from 19 to 31 years $(M=23.5)$, and all were right-handed by self-report.

\section{Apparatus and stimuli}

The apparatus was the same as in the previous experiment. The digits $1,2,3,4,6,7,8,9$ and the letters $A, E, I, U, G$, K, M, R served as stimuli. The first stimulus always appeared laterally to the left or right of the center of the screen at an eccentricity of $1.95^{\circ}$. The second stimulus was always presented centrally

\section{Procedure}

The participants had to judge the parity (odd or even) or the letter type (consonant or vowel) of the presented numerals or letters, respectively. The required responses to the first and second stimulus were the same as in the previous experiment. The categories "even" and "consonant" were mapped to the left response keys on each response box, and "odd" and "vowel" to the right response keys.

The temporal order of presentation on a given trial was also the same as in Experiment 1. As task cues, which were presented at the center of the screen, served "g/ $u$ " (for the parity judgments) and " $\mathrm{k} / \mathrm{v}$ " (abbreviations for the German words "Konsonant" (consonant) and "Vokal" (vowel) for the letter type judgment). As in the first experiment, cue identity always indicated the judgment type required for the first stimulus, and the color of the cue (green or red) indicated whether the same or the other task had to be performed for the second stimulus. Notice that the cues were completely redundant in this experiment, since the stimuli were univalent (i.e. afforded only one of the two possible tasks).

In total, the participants performed 31 blocks of 60 trials each in three 1-h sessions. The first three blocks in the first session, and the first two blocks in the second and third session were considered as practice and were not analyzed.

\section{Results}

The latencies and error rates for the first response were analyzed by separate two-way repeated measures ANOVAs, considering the factors task (repetition, switch) and 
compatibility (compatible, incompatible) of the first stimulus. Regarding the response times and error rates for the second response, the factors task (repetition, switch), compatibility of the first stimulus (compatible, incompatible), and response (repetition, shift) were considered in separate three-way repeated measures ANOVAs.

\section{Responses to the first stimulus}

$R T$. The analysis revealed a significant main effect of task, $F(1,11)=16.01, P<0.01$, which reflects slower responses on task switch trials than on task repetition trials (537 vs. $530 \mathrm{~ms}$ ). Also, the main effect of compatibility was reliable, $F(1,11)=6.00, P<0.05$, reflecting faster responses for compatible than for incompatible stimuli ( 529 vs. $537 \mathrm{~ms}$ ).

$E R$. The overall mean error rate was rather low $(2.88 \%)$ and there were no significant effects.

\section{Responses to the second stimulus}

The RTs and ERs for the second response on a trial are given in Table 2 .

$R T$. The analysis revealed a significant main effect of task, $F(1,11)=7.62, P<0.05$, reflecting task switch costs of $32 \mathrm{~ms}$. Furthermore, the interaction of compatibility and response was significant, $F(1,11)=5.60, P<0.05$. As can be seen in Fig. 3, response repetitions produced larger overall costs if the first stimulus was compatible than if it was incompatible.

$E R$. The analysis revealed significant main effects of task, $F(1,11)=5.50, \quad P<0.05$, and of response, $F(1,11)=9.12, P<0.01$. As expected, task repetitions produced lesser errors than task switches (1.90 vs. $3.23 \%$ ). Response repetitions, though, were disadvantageous relative to response shifts ( 2.95 vs. $2.19 \%$ ). Furthermore, the interaction between compatibility and response was significant, $F(1,11)=13.86, P<0.01$. As Fig. 3 shows, there were larger costs for response repetitions if the first stimulus was compatible $(1.32 \%)$ than if it was incompatible $(0.20 \%)$

Table 2 Response times (in $m s$ ) and error rates (in $\%$, in parentheses) for the second response on a trial in Experiment 2, depending on task, response, and $S R$-compatibility of the first stimulus

\begin{tabular}{llllll}
\hline & \multicolumn{2}{l}{ Task repetition } & & \multicolumn{2}{l}{ Task switch } \\
\cline { 2 - 3 } \cline { 5 - 6 } & RCR & RCS & & RCR & RCS \\
\hline Compatible & $502(2.20)$ & $508(1.78)$ & & $548(4.43)$ & $526(2.21)$ \\
Incompatible & $496(1.56)$ & $507(2.08)$ & & $537(3.60)$ & $528(2.69)$
\end{tabular}

$\overrightarrow{R C R}$ response category repetition, $R C S$ response category shift

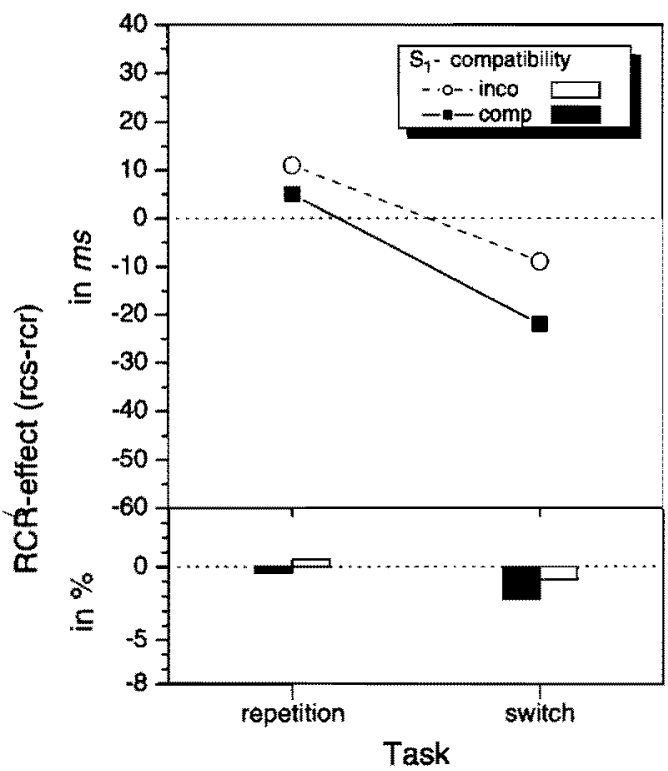

Fig. 3 Response category repetition effects in Experiment 2, depending on the SR-compatibility of the first stimulus and on the task condition. In the legend: comp compatible, inco incompatible, rer response category repetition, res response category shift

\section{Discussion}

In the present experiment we modulated response activation by means of SR-compatibility. As expected, performance was better (i.e. faster responses and less errors) when the stimuli appeared on the same side as the required response than when they appeared at the opposite side. This indicates that the responses were indeed more activated for compatible stimuli than for incompatible ones. Most important for the present objective, though, this modulation also affected the response repetition effects. On task switch trials, the response repetition costs were larger for compatible first stimuli than for incompatible first stimuli. Likewise, on task repetition trials, the response repetition benefits were smaller for compatible compared to incompatible first stimuli. This indicates that the degree of response inhibition merely depends on the amount of the current (relative) response activation, but not on the source of the activation. That is, it does not matter whether the responses are additionally activated by congruency as in Experiment 1, or by SR-compatibility as in the present experiment.

The result that we observed reliable repetition costs for univalent stimuli is different from the results in our previous study (Hübner \& Druey, 2006). In that study, response repetition costs occurred only for bivalent stimuli. One reason for this difference might be that in our previous study, task repetitions and task switches were blocked, 
whereas they were randomized in the present experiment. However, it is more likely that the costs are a result of the lateral stimulus presentation: It is reasonable to assume that the compatibility of the first stimulus increased the risk of an accidental re-execution of the last response in an analogous way as the congruency with bivalent stimuli. The fact, however, that the second stimulus always appeared centrally, reduced the risk of accidental response re-executions again compared to the first experiment, where also the second stimulus was either congruent or incongruent. Taken together, these aspects of the present procedure might not only explain why we also observed costs for response repetitions for univalent stimuli in the present experiment, but also why no such costs appeared on task repetition trials, even though the timing of presentation was the same as in the previous experiment. In other words, although there was speed stress in the present experiment also, the resulting response inhibition was presumably smaller due to the presentation of univalent stimuli.

\section{Experiment 3}

In the first two experiments we have shown that the (relative) activation of a response determines the degree of its inhibition, irrespective of whether this activation is modulated by means of congruency or SR-compatibility. Therefore, the aim of the present experiment was to investigate how these two sources of response activation act together. In order to achieve this aim, we again used bivalent stimuli (digits) as in Experiment 1. This time, however, the first stimulus always appeared laterally as in Experiment 2. The question was whether congruency and SR-compatibility affect the response repetition effects in an additive or in an interactive way. Given the PDP models mentioned in the "Introduction" (e.g. Botvinick et al,, 2001; Gilbert \& Shallice, 2002), one would expect that the activations from the two sources were added to determine the net activation of the respective response. Consequently, the effects for congruency and SR-compatibility in response to the first stimulus should be additive. Moreover, the same should hold for the modulation of the repetition effects in response to the second stimulus, if the strength of response inhibition depends linearly on the strength of the previous response activation. It is, however, also reasonable to assume that response inhibition may be limited so that a simultaneous response activation from two sources leads to weaker inhibition than would have been expected from the sum of the activation of the two sources. If this were the case, then congruency and SR-compatibility should produce underadditive effects on the repetition effects in the second task.

\section{Method}

\section{Participants and stimuli}

Twelve students (nine female, three male) participated in this experiment under the same conditions as in Experiments 1 and 2 . Their age ranged from 20 to 25 years $(M=22.7)$ and all were right-handed by self-report.

The same digits as in the previous experiments served as stimuli.

\section{Procedure}

$-$

As in the first experiment, the participants had to judge the parity (odd or even) or the magnitude (less or greater than 5) of the presented numerals on four keys, two for each hand.

The SR-mapping, the temporal order of presentation, and the cueing procedure were also the same as in Experiment 1. However, as in Experiment 2, the first stimulus always appeared laterally to the left or right of central fixation.

In total, the participants performed 66 blocks of 32 trials each in three 1 -h sessions. The first four blocks in the first session, the first three blocks in the second session and the first two blocks in the third session were considered as practice and were not analyzed.

\section{Results}

For the responses to the first stimulus, the factors task (repetition, switch), compatibility (compatible, incompatible), and congruency (congruent, incongruent) were analyzed separately for response times and error rates by three-way repeated measures ANOVAs. Analogous ANOVAs were computed for the responses to the second stimulus, with the factors task (repetition, switch), compatibility of the first stimulus (compatible, incompatible), congruency of the first stimulus (congruent, incongruent), and response (repetition, shift).

\section{Responses to the first stimulus}

The response times and error rates for the responses to the first stimulus are given in Table 3 .

$R T$. The analysis revealed a significant main effect of task, $F(1,11)=6.91, P<0.05$, reflecting that the response times were increased on task switch trials relative to task repetition trials. Furthermore, the main effects of compatibility, $F(1,11)=9.01, P<0.05$, and of congruency, $F(1,11)=9.56, P<0.05$, were reliable. Responses to compatible stimuli were faster than those to incompatible stimuli. An analogous relation holds for congruency. The 
Table 3 Response times (in $m s$ ) and error rates (in \%; in parentheses) for the response to the first stimulus in Experiment 3, depending on task, congruency, and SR-compatibility

\begin{tabular}{llllll}
\hline & \multicolumn{3}{c}{ Task repetition } & \multicolumn{2}{l}{ Task switch } \\
\cline { 2 - 3 } \cline { 5 - 6 } & Congruent & Incongruent & Congruent & Incongruent \\
\hline Compatible & $418(2.12)$ & $441(5.54)$ & $431(2.27)$ & $457(4.54)$ \\
Incompatible & $425(2.31)$ & $449(6.58)$ & $441(2.31)$ & $466(6.85)$ \\
\hline
\end{tabular}

interaction between compatibility and congruency was not reliable $(F(1,11)<1, P>0.88)$.

$E R$. Regarding the error rates, the analysis revealed significant main effects of compatibility, $F(1,11)=5.54$, $P<0.05$, and of congruency, $F(1,11)=18.13, P<0.01$. However, there was also a significant interaction between these two factors, $F(1,11)=8.13, P<0.05$. For congruent stimuli, it did not matter whether they were also compatible or incompatible. However, for incongruent stimuli, more errors occurred if they were also incompatible than if they were compatible.

\section{Responses to the second stimulus}

The response times and error rates for the second response on a trial are given in Table 4.

$R T$. The analysis of the responses to the second stimulus revealed a reliable main effect of task, $F(1,11)=10.25$, $P<0.01$, reflecting task switch costs of $14 \mathrm{~ms}$. However, this effect was qualified by a reliable interaction between task and response, $F(1,11)=17.34, P<0.01$. Response repetitions produced benefits of $14 \mathrm{~ms}$ on task repetition trials, but costs of $21 \mathrm{~ms}$ on task switch trials. Furthermore, the two-way interactions between compatibility and response, $F(1,11)=5.38, P<0.05$, and between congruency and response, $F(1,11)=10.61, P<0,01$, were significant. As can be seen in Fig. 4, both compatible and congruent first stimuli shifted the response repetition effects in the direction of (larger) costs on task repetition as well as on task switch trials. The three-way interaction between compatibility of the first stimulus, congruency of the first stimulus, and response was not reliable $(F<1.4, P>0.27)$.

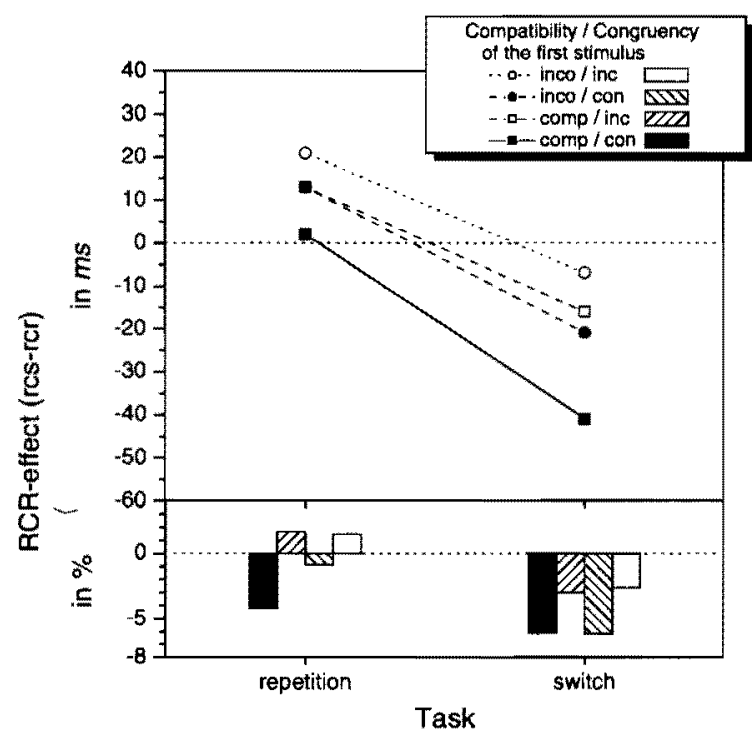

Fig. 4 Response category repetition effects in Experiment 3, depending on the SR-compatibility and the congruency of the first stimulus, and on the task condition. In the legend: comp compatible, con congruent, inco incompatible, inc incongruent, rcr response category repetition, res response category shift

$E R$. The analysis of the error rates revealed significant main effects of task, $F(1,11)=12.69, P<0.01$, and of response, $F(1,11)=17.43, P<0.01$. However, there was also a reliable interaction between these two factors, $F(1,11)=17.12, P<0.01$, reflecting that on task switch trials there were larger response repetition costs than on task repetition trials $(4.51$ vs. $0.48 \%)$. Furthermore, the interaction between task and compatibility was significant, $F(1,11)=8.49, \quad P<0.05$. The participants made more errors on task repetition trials if the first stimulus on a trial was compatible compared to when it was incompatible. This pattern was reversed on task switch trials. Also the main effect of congruency was significant, $F(1,11)=7.69$, $P<0.05$, as was the two-way interaction between response and congruency, $F(1,11)=36.75, P<0.001$. The interaction is due to the fact that after a congruent first stimulus, erroneous response shifts occurred more often than erroneous

Table 4 Response times (in $m s$ ) and error rates (in $\%$; in parentheses) for the second response in Experiment 3, depending on task, response, congruency of the first stimulus, and SR-compatibility of the first stimulus

\begin{tabular}{|c|c|c|c|c|c|}
\hline & & \multicolumn{2}{|c|}{ Task repetition } & \multicolumn{2}{|l|}{ Task switch } \\
\hline & & $\mathrm{RCR}$ & $\mathrm{RCS}$ & $\mathrm{RCR}$ & $\mathrm{RCS}$ \\
\hline \multirow[t]{2}{*}{ Congruent } & Compatible & $419(8.16)$ & $421(3.97)$ & $458(10.59)$ & $417(4.43)$ \\
\hline & Incompatible & $413(5.30)$ & $429(4,43)$ & $446(9.90)$ & $425(3.71)$ \\
\hline \multirow[t]{2}{*}{ Incongruent } & Compatible & $413(4.34)$ & $429(5.99)$ & $439(7.10)$ & $423(4,08)$ \\
\hline & Incompatible & $413(3.82)$ & $434(5.27)$ & $439(7.88)$ & $432(5.21)$ \\
\hline
\end{tabular}

$R C R$ response category repetition, $R C S$ response category shift 
response repetitions. After an incongruent first stimulus, this difference was less pronounced. The three-way interaction between response, congruency, and compatibility was not reliable $(F=1.1, P=0.33)$

\section{Discussion}

After we have shown in the first two experiments that SRcompatibility and congruency affect the response repetition effects on a subsequent trial in a similar manner, the aim of the present experiment was to investigate their common effects. First of all, if we consider the responses to the first stimulus, then both compatibility and congruency had a significant effect (cf. Meiran, 2005, for a similar result in a spatial task-switching paradigm) ${ }^{2}$. This shows that a response can simultaneously and independently be activated by different task irrelevant features. Most important for the present objective, both congruency and compatibility of the first stimulus also affected the response repetition effects for the second response. Statistically, the effects were even additive. However, because we had only 12 participants, one might argue that the power to detect an interaction was relatively small. If we consider the data in detail, though, then it is obvious that there was a tendency towards overadditivity (at least on task switch trials). Consequently, we can at least rule out that our manipulations impinge on some limit of response inhibition. It thus seems justified to conclude that the strength of response inhibition is directly and (almost) linearly determined by the amount of response activation, irrespective of the sources of this activation.

\section{General discussion}

In several previous studies it has been shown that response repetitions produce benefits on task repetition trials, but costs on task switch trials (e.g., Hübner \& Druey, 2006; Schuch \& Koch, 2004; Kleinsorge, 1999; Meiran, 2000a; Rogers \& Monsell, 1995). In one of our own studies (Hübner \& Druey, 2006), we already provided some evidence that response inhibition plays a crucial role in explaining this interaction. We assumed that a response is generally inhibited after it has been activated in order to prevent its accidental re-execution on the next trial (cf., e.g., Logan \& Gordon, 2001; Smith, 1968). That response repetition

\footnotetext{
${ }^{2}$ For the response times, the effects of congruency and SR-compatibility were additive, as could have been expected from the PDP models mentioned in the introduction (Botvinick et al., 2001; Gilbert \& Shallice, 2002; Houghton \& Tipper, 1994). With respect to the error rates, however, this was not the case. The reason for this interaction might though simply reflect a floor effect: Given a congruent stimulus, errors are already so infrequent that even if the stimulus was also compatible, this could not further improve performance.
}

benefits occur on task repetition trials merely reflects the fact that on these trials also the stimulus category (e.g., "odd") repeats and that the positive effects of this repetition outweigh the negative inhibition effects. Because stimulus category repetitions are absent on task switch trials, this condition seems to be most suitable for the examination of pure response inhibition effects.

In the present series of experiments, we examined further details of this inhibition account. Specifically, we were interested in factors determining the strength of response inhibition. It seems reasonable to assume that the strength of inhibition depends on the risk of an erroneous response re-execution and that one modulating factor of this risk is the residual response activation left over from the previous trial (cf. Hübner \& Druey, 2006).

One way to test this idea would be to modulate the response activation and to observe whether the inhibition varies accordingly. In our previous study, we did this by presenting stimuli which were either congruent or incongruent. Because congruent stimuli not only activate the correct response according to the required task, but also according to the irrelevant task, one may suspect that the (relative) response activation is increased for these stimuli compared to incongruent stimuli (cf., e.g., Gilbert \& Shallice, 2002). If this variation affects response inhibition in the expected way, then response repetition costs on task switch trials should be larger if the stimulus on the previous trial was congruent rather than incongruent. Indeed, we observed such an effect (Hübner \& Druey, 2006). However, the generality of this result is limited, because, in that study, we used a dual-task procedure with temporally overlapping tasks. Therefore, the aim of the first experiment in the present study was to replicate this finding with a procedure that was more similar to that applied in common task switch studies.

As a result, despite the different procedure, the response repetition costs on switch trials were again larger if the first stimulus on a trial was congruent rather than incongruent. Interestingly, even on task repetition trials there were response repetition costs if the first stimulus was congruent. Only for incongruent stimuli on task repetition trials, response repetition benefits could be observed. Altogether, the effects observed in Experiment 1 are a replication and generalization of our former results.

In Experiment 2, it was investigated whether it is crucial that the modulation of the response activation is produced by a stimulus category, which is generally relevant within the experimental context or not. Therefore, we used univalent stimuli (digits and letters) and modulated response activation by always presenting the first stimulus laterally to the left or right of central fixation, which was completely irrelevant for the tasks at hand. Because the tasks required a "left" or "right" response, the stimuli were either spatially 
compatible or incompatible (e.g. Simon, 1969; Hommel, 1993; see Lu \& Proctor, 1995, for an overview). As expected, responses to compatible stimuli were faster than those to incompatible ones. Most important for the present objective, though, the response repetition costs on task switch trials were larger when the stimulus on the previous trial was spatially compatible rather than incompatible. Analogously, the response repetition benefits on task repetition trials were reduced. These effects indicate that it does not matter for the modulation of the response repetition effects whether the additional response activation is caused by a task-related or by a task-unrelated stimulus feature.

In Experiment 3, we then investigated how the two sources of additional response activation used in the first two experiments act together. As a result, the variations had additive effects. This suggests that the strength of response inhibition linearly depends on the amount of previous response activation. In other words, at least the fact that we did not observe underadditive effects (but rather a weak tendency towards overadditivity) rules out that response inhibition is limited. Thus, our results indicate that the number of sources and their origin seem to be irrelevant with respect to (the strength of) response inhibition.

Altogether, the results of the three experiments demonstrate that the size of the response repetition effects depends on the (relative) amount of response activation on the previous trial. Furthermore, since an increased response activation produced larger response repetition costs on task switch trials and less benefits on task repetition trials, the results strongly support our response inhibition account (Hübner \& Druey, 2006, 2007).

Even though response inhibition properly accounts for our results, one might ask whether the proposed alternative explanations could also account for our data. As mentioned, according to the reconfiguration account (e.g, Kleinsorge, 1999), a task switch induces a tendency to also shift the response. Although such an assumption would indeed explain response repetition costs on task switch trials, it fails to predict any dependence of the size of the costs on the response activation on the previous trial. The same holds for the modulation of the response repetition benefits on task repetition trials.

According to the strengthening account (e.g. Meiran, 2000a; Schuch \& Koch, 2004), the association between the response and the stimulus category for the relevant task is strengthened, whereas that between the response and the stimulus category of the irrelevant task is weakened after responding. Accordingly, if the task repeats, and the last response is required again, the association between the relevant stimulus category and the response is in a strengthened state, which explains the response repetition benefits. In case the task switches though, the association between the last response and the currently relevant stimulus category is in a weakened state, which explains the response repetition costs. However, similar to the reconfiguration hypothesis, this account also fails to explain why response repetition effects depend on the degree of the previous (relative) response activation. Even if one additionally assumes that (a) the strength of the relevant category-response association depends on the amount of activation of the involved stimulus category and response, as is supposed in several of the already mentioned PDP models (e.g. Botvinick et al, 2001; Gilbert \& Shallice, 2002), and (b) the strength of the irrelevant category-response association depends on the strength of the relevant category-response association, this would merely account for the increased response repetition costs on task switch trials after a congruent or compatible stimulus. The corresponding decreased benefits on task repetition trials, however, could not be explained by such a model. Rather, the assumption of an increased association strength would even predict increased response repetition benefits after congruent or compatible stimuli on task repetition trials, which is opposite to our results.

Although it is principally possible to integrate our response inhibition account and the strengthening accounts, so that the inhibition of response categories explains the modulations of the repetition effects on task repetition and task switch trials depending on the previous activation, and the strengthening and weakening of category-response associations accounts for the basic interaction between task sequence and response sequence, this would lead to a rather complex account for our results. The question is why one should prefer such a complex account, if it is sufficient to assume simple activation and inhibition mechanisms for the different types of categories (i.e. stimulus and response categories) involved in performing such tasks as in the present study. In order to also explain task switch costs though, it might nevertheless be necessary to adopt the more complex integrated model. Therefore, in the next section, we will shortly discuss a recently presented model, which in our view seems particularly appropriate for an integration of the strengthening and inhibition principles.

\section{Sequential control in task switching}

Recently, there has been an increased interest in the area of mental control and how such control is brought about (e.g. Botvinick et al., 2001; Egner \& Hirsch, 2005). Brown et al, (2007), for instance, attempted to fractionate different aspects of control in task switching. Although they were primarily interested in higher-order sequential effects (i.e. effects over the run of more than two trials), they also addressed how the congruency of the stimuli on previous trials affects performance on the present trial. Moreover, they also assumed that control might especially be relevant "to prevent an anticipated response from being prematurely 
(and erroneously) generated before external stimuli can be adequately processed" (p. 41). However, contrary to our assumption, they proposed a PDP-model, in which the interaction of task sequence and response sequence is conceptualized in terms of the strengthening and weakening of associations (cf. Meiran, 2000a, b; Schuch \& Koch, 2004). Thus, despite relying on similar underlying principles, this model would not predict our present results. Nevertheless, the model is highly interesting, since it integrates several previously isolated findings from different task switch studies. Thus, if the model would be expanded to also include a response (self-) inhibition mechanism (cf. Houghton \& Tipper, 1994), it might provide a comprehensive explanation of the different effects observed in task switch studies.

\section{Anticipatory task switch costs in response to the first stimulus}

One interesting finding additional to the repetition effects described so far concerns the fact that we observed task switch costs also in response to the first stimulus (see also Hübner \& Druey, 2006). Given the procedures in the present (and also in our previous) study, these costs are not surprising though. Whereas in our previous study, the participants knew in advance whether a task switch or a task repetition was required since we blocked the type of task transition, this varied randomly from trial to trial in the present study. Nevertheless, due to the fact that, at the beginning of each trial, we cued whether a task repetition or a task switch was required with the second stimulus relative to the first one, the participants in this study also knew in advance whether the task would repeat or switch. Thus, actually we combined two different cueing procedures in the present study: for the first stimulus the required task was cued directly. For the second stimulus, the task was cued relative to the first task, which means that we actually used a special type of transition cue (Forstmann, Brass, \& Koch, 2007). If the participants indeed use the presented cue in order to prepare for the upcoming tasks, as is indicated by various results showing that preparation reduces the task switch costs (e.g. De Jong, 2000; Rogers \& Monsell, 1995) then it would not be surprising if the simultaneous preparation of two tasks produces costs even in a first, directly cued task (cf. Logan \& Gordon, 2001).

However, with respect to the response repetition effects, we would not expect that this cueing procedure matters. In their study, Forstmann et al. (2007) already showed that the basic pattern of task switch-related effects is the same irrespective of whether direct cues or transition cues are used. The restrictions they discussed with respect to their specific procedure (e.g., the fact that with typical transition cues, three-task sequences rather than two-task sequences are considered) does not hold for the present study, since we combined direct and indirect cueing in our two-task sequencing procedure.

\section{Conclusion}

The results of the present experiments provide further evidence in favor of an inhibition account for the response repetition effects under task switch conditions, as we could show that the strength of the inhibition of the just performed response depends on the strength of its previous activation. Moreover, simple activation and inhibition mechanisms are sufficient to explain a large range of response repetition effects in various tasks.

Acknowledgments We are grateful to Nachshon Meiran, Iring Koch, and an anonymous reviewer for their helpful comments on a previous version of this manuscript. Furthermore, we thank Johannes Schult and Karen Donath for collecting the data. This research was supported by a grant ( $\mathrm{Hu} 432 / 9)$ from the Deutsche Forschungsgemeinschaft (DFG) to the second author

\section{References}

Botvinick, M. M., Braver, T. S., Barch, D. M., Carter, C. S., \& Cohen, J. D. (2001). Conflict monitoring and cognitive control. Psychological Review, 108, 624-652.

Brown, J. W., Reynolds, J. R., \& Braver, T. S. (2007). A computational model of fractionated conflict-control mechanisms in task-switching. Cognitive Psychology, 55, 37-85.

Campbell, K. C., \& Proctor, R. W. (1993). Repetition effects with categorizable stimulus and response sets. Journal of Experimental Psychology: Learning, Memory, and Cognition, 19, 1345-1362.

De Jong, R. (2000). An intention-activation account of residual switch costs. In S. Monsell, J. Driver (Eds.), Attention and performance XVIII: Control of cognitive processes (pp. 357-376). Cambridge: MIT.

De Jong, R., Liang, C. C., \& Lauber, E. (1994). Conditional and unconditional automaticity: A dual-process model of effects of spatial stimulus-response correspondence. Journal of Experimental Psychology: Human Perception and Performance, 20, 731-750.

Egner, T., \& Hirsch, J. (2005). Cognitive control mechanisms resolve conflict through cortical amplification of task-relevant information. Nature Neuroscience, 8, 1784-1790.

Eimer, M. (1995). Stimulus-response compatibility and automatic response activation: Evidence from psychophysiological studies. Journal of Experimental Psychology: Human Perception and Performance, 21, 837-854.

Forstmann, B. U., Brass, M., \& Koch, I. (2007). Methodological and empirical issues when dissociating cue-related from task-related processes in the explicit task-cuing procedure. Psychological Research, 71, 393-400.

Gilbert, S. J., \& Shallice, T. (2002). Task switching: A PDP model. Cognitive Psychology, 44, 297-337.

Hommel, B. (1993). Inverting the Simon effect by intention: Determinants of direction and extent of effects of irrelevant spatial information. Psychological Research, 55, 270-279.

Hommel, B. (1995). Stimulus-response compatibility and the Simon effect: Toward an empirical clarification. Journal of Experimental Psychology: Human Perception and Performance, 21, 764-775. 
Hommel, B. (1998). Event files: Evidence for automatic integration of stimulus-response episodes. Visual Cognition, 5, 183-216.

Houghton, G., \& Tipper, S. P. (1994). A model of inhibitory mechanisms in selective attention. In D. Dagenbach, T. H. Carr (Eds.), Inhibitory processes in attention, memory, and language (pp. 53112). San Diego: Academic.

Hübner, R., \& Druey, M. D. (2006). Response execution, selection, or activation: What is sufficient for response-related repetition effects under task shifting? Psychological Research, 70, 245-261.

Hübner, R., \& Druey, M. D. (2007). Multiple response codes play specific roles in response selection and inhibition under task shifting. Psychological Research. (in press).

Kleinsorge, T. (1999). Response repetition benefits and costs. Acta Psychologica, 103, 295-310.

Kornblum, S., Hasbroucq, T., \& Osman, A. (1990). Dimensional overlap: Cognitive basis for stimulus-response compatibility-a model and taxonomy. Psychological Review, 97, 253-270.

Logan, G. D., \& Gordon, R. D. (2001). Executive control of visual attention in dual-task situations. Psychological Review, 108, 393434.

Lu, C. H., \& Proctor, R. W. (1995). The influence of irrelevant location information on performance: A review of the Simon and spatial Stroop effects. Psychonomic Bulletin \& Review, 2, 174-207.

Mayr, U., \& Kliegl, R. (2003). Differential effects of cue changes and task changes on task-set selection costs. Journal of Experimental Psychology: Learning, Memory, and Cognition, 29, 362-372.

Meiran, N. (2000a). Modeling cognitive control in task-switching. Psychological Research, 63, 234-249.

Meiran, N. (2000b). Reconfiguration of stimulus task sets and response task sets during task switching. In S. Monsell, J. Driver (Eds.), Attention and performance XVIII: Control of cognitive processes (pp. 377-399). Cambridge: MIT.

Meiran N. (2005). Task rule-congruency and Simon-like effects in switching between spatial tasks. The Quarterly Journal of Experimental Psychology, 58A, 1023-1041.

Meiran N., Chorev Z., \& Sapir, A. (2000). Component processes in task switching. Cognitive Psychology, 41, 211-253.

Notebaert W., \& Soetens, E. (2003). The influence of irrelevant stimulus changes on stimulus and response repetition effects. Acta Psychologica, 112, 143-156.

Notebaert W., Soetens E., \& Melis, A. (2001). Sequential analysis of a Simon task-evidence for an attention-shift account. Psychological Research, 65, 170-184.
Pashler H., \& Baylis, G. (1991). Procedural learning: 2. Intertrial repetition effects in speeded-choice tasks. Journal of Experimental Psychology: Learning, Memory, and Cognition, 17, 33-48.

Ridderinkhof, K. R. (1997). A dual-route processing architecture for stimulus-response correspondence effects. In B. Hommel, W. Prinz (Eds.), Theoretical issues in stimulus-response compatibility (pp. 119-131). Amsterdam: Elsevier.

Ridderinkhof, K. R. (2002). Micro- and macro-adjustments of task set: Activation and suppression in conflict tasks. Psychological Research, 66, 312-323.

Rogers, R. D., \& Monsell, S. (1995). Costs of a predictable switch between simple cognitive tasks. Journal of Experimental Psychology: General, 124, 207-231.

Schuch, S., \& Koch, I. (2004). The costs of changing the representation of action: Response repetition and response-response compatibility-in dual tasks. Journal of Experimental Psychology: Human Perception and Performance, 30, 566-582.

Simon, J. R. (1969). Reactions toward the source of stimulation. Journal of Experimental Psychology, 81, 174-176.

Smith, M. C. (1968). Repetition effect and short-term memory. Journal of Experimental Psychology, 77, 435-439.

Soetens, E. (1998). Localizing sequential effects in serial choice reaction time with the information reduction procedure. Journal of Experimental Psychology: Human Perception and Performance, $24,547-568$.

Soetens, E., Melis, A., \& Notebaert, W. (2004). Sequence learning and sequential effects. Psychological Research, 69, 124-137.

Steinhauser, M., \& Hübner, R. (2006). Response-based strengthening in task shifting: Evidence from shift effects produced by errors. Journal of Experimental Psychology: Human Perception and Performance, 32, 517-534.

Stürmer, B., Leuthold, H., Soetens, E., Schroeter, H., \& Sommer, W, (2002). Control over location-based response activation in the Simon task: Behavioral and electrophysiological evidence. Joumal of Experimental Psychology: Human Perception and Performance, 28, 1345-1363.

Stürmer, B., Seiss, E., \& Leuthold, H. (2005). Executive control in the Simon task: A dual-task examination of response priming and its suppression. European Journal of Cognitive Psychology, 17 $590-618$.

Wühr, P., \& Ansorge, U. (2005). Exploring trial-by-trial modulations of the Simon effect. The Quarterly Journal of Experimental Psychology, 58A, 705-731. 